Thorax (1964), 19, 37

\title{
Bronchography with Hytrast
}

\author{
B. T. LE ROUX AND J. G. DUNCAN \\ From the Thoracic Surgical and Radiodiagnostic Departments, The Royal Infirmary, Edinburgh
}

Contrast media which outline tube systems are well established radiodiagnostic adjuncts. Techniques and media may be changed both to increase the margin of safety for the patient and to improve radiographic contrast. In the radiographic demonstration of bronchi, early techniques, such as the insufflation of barium powder through a bronchoscope (Jackson, 1918) and the instillation of oily suspensions of bismuth, were quickly discarded as dangerous. Iodized poppy-seed oil (Lipiodol) was the first practical bronchographic agent (Sicard and Forestier, 1922) and was the only medium in general use for many years. The main disadvantage of Lipiodol was its long retention in pulmonary parenchyma in a radio-opaque form, especially where alveolar spill had occurred, so that the interpretation of serial radiographs used to demonstrate the progress of a pulmonary lesion was complicated. A granulomatous reaction to the oil was also an occasional occurrence.

Dormer (1940) used Lipiodol as a vehicle for the introduction of sulphanilamide powder into the bronchi as a topical therapeutic agent in the control of bronchial infection. The sulphonamide increased the viscosity of the iodized oil, and it was soon recognized that by its addition alveolar spill was diminished, contrast was greater because the oil was less widely dispersed, and more complete post-bronchographic evacuation of the contrast medium was achieved by coughing, so that subsequent chest radiographs commemorated less frequently and grossly previous bronchographic attempts. The combination of $8 \mathrm{~g}$. of sulphanilamide powder with $20 \mathrm{ml}$. of Lipiodol was used for bronchography in the Thoracic Surgical Unit in Edinburgh between 1950 and 1955. Some 800 bronchograms were made. Two patients experienced such severe dyspnoea as a consequence of bronchial obstruction by the viscous material that they very nearly suffocated, and in a third patient the subsequent development of acute nephritis was related to bronchography. Other complications were not encountered, and $75 \%$ of the bronchograms produced were then regarded as satisfactory. In a more critical retrospective evaluation of these bronchograms five years later (le Roux, 1962), it was thought that there was justification for dissatisfaction in $45 \%$ rather than $25 \%$ of the bronchograms. Improvement in bronchographic technique had made unacceptable that which in the past had been regarded as adequate. With more general use of the combination of sulphonamide with Lipiodol, marketed as Visciodol (Burrascano, 1955 ; Johnson and Irwin, 1959), the hazards of sensitivity to sulphonamide, of the formation of methaemoglobin, and of widespread bronchial obstruction by a too viscid preparation became apparent.

In the attempt to obviate these disadvantages, water-soluble media were prepared. The first of these was diodone, combined with a thickening agent such as carboxymethylcellulose or polyvinyl pyrrolidone. This contrast medium proved clinically unacceptable because it was excessively irritating to the bronchi. In 1953 a new compound, propyliodone, was described. It was originally introduced as an aqueous suspension (Dionosil Aqueous) for which the advantages of rapid disappearance from the lungs and relative freedom from the risks of iodism were claimed. Its use was, however, often followed by a febrile reaction in the patient and, although isotonic with body fluids, it also proved irritating to the bronchial mucosa. The hazards of local anaesthetic sideeffects were enhanced because of the need to suppress more effectively the cough reflex with larger doses of topical anaesthetic. Propyliodone in arachis oil (Dionosil Oily) was then introduced as an alternative to the aqueous suspension, and this was shown to be less irritating and, on serial radiographs, was rarely retained in the bronchi or alveoli for longer than 48 hours. In Edinburgh after 1956 the oily suspension of propyliodone was adopted almost routinely as a bronchographic medium. While radiographic contrast was often less effective than with sulphanilamide powder in iodized poppy-seed oil, reactions were not observed, and the standard of bronchography was 
regarded as adequate. When bronchography was followed by pulmonary resection, granulomatous reaction to the oily vehicle was not observed.

In 1957 a technique of bronchography in children was developed (le Roux and Masson, 1962) which, since 1961, has been adopted for all patients, children and adults, who require bronchography. This technique depends on general anaesthesia with sodium thiopentone and a relaxant (suxamethonium). After induction of anaesthesia and inflation of the lungs with oxygen delivered through a face-piece, the bronchi are examined under direct vision. On completion of bronchoscopy the lungs are again inflated with oxygen, and a cuffed endotracheal tube is introduced under direct vision into the trachea. The cuff of the tube is inflated and the endotracheal tube connected to a Boyle or similar machine. Inflation is continued with oxygen or oxygen and nitrous oxide. The patient is then turned on his right side, lies on an $x$-ray cassette, and the $x$-ray tube anode is positioned. A catheter is passed through the endotracheal tube and, from a $20 \mathrm{ml}$. syringe, the contrast medium is injected rapidly into the trachea. The rubber bung of the Magill T-piece is replaced, and the lungs are inflated for five breaths. Inflation is forceful since it is by pulmonary inflation that the contrast medium is blown into the dependent lung. The radiographic plate for the right lateral bronchogram is then exposed, the patient turned on to his back, a second cassette placed under him, and an anteroposterior view of the right side made. During the moment of radiographic exposure the anaesthetist holds the lungs inflated to depress the domes of the diaphragm. The same procedure is then adopted for the left side, where an oblique and not a lateral view is made. The entire procedure, including bronchoscopy, occupies usually less than five minutes, and it is rarely necessary to inject a supplementary dose of relaxant. The anaesthetist has full control of ventilation except during bronchoscopy, and the technique of bronchography by insufflation, as opposed to a technique which demands changes in posture, is time-saving. Tracheo-bronchial toilet at the end of the procedure usually results in the elimination by suction of a considerable volume of the contrast medium. Another feature of this technique of bronchography is that it has removed from the investigation all element of mystique. The need for positioning the patient at a number of arbitrary angles for arbitrary periods of time lent, for the uninitiated, an air of profundity to the experienced bronchographer. And there are no longer those moments of suspense while the radiographs are being developed and before the scrutiny of which it was not known how complete a bronchogram had been achieved or whether only an outline of the oesophagus would be visible. The insufflation technique is precisely definable in terms of position, either right or left lateral, and number of pulmonary insufflations with the patient paralysed. This technique produced uniformly satisfactory bronchograms with the oily suspension of Dionosil. More than 500 bronchograms were made and fewer than $2 \%$ were inadequate for reaching a decision on management of the patient.

At the end of 1962 the Thoracic Surgical Unit and the Department of Diagnostic Radiology at the Royal Infirmary in Edinburgh were asked to assess the value of Hytrast ${ }^{1}$ as a bronchographic medium. Hytrast is an opaque white suspension with a $p \mathbf{H}$ of about 7 , obtained by the dispersion of a mixture of fine crystals (2-5 microns) of N(propyl-2:3-diol)-3:5 diiodo-4-pyridone and 3:5-diiodo-4-pyridone in a slightly hypotonic aqueous solution of sodium carboxymethylcellulose. These compounds differ from propyliodone and diodone in that they are not esters and do not have labile functional groupings. Esters hydrolyse in the bronchi and produce acidic ions which are thought to be responsible for the local irritant properties of diodone and propyliodone. The iodine concentration in Hytrast $(0.5 \mathrm{~g} . / \mathrm{ml}$. $)$ is greater than in propyliodone $(0.28 \mathrm{~g} . / \mathrm{ml}$.) so that radiographic contrast should be increased, and, being chemically relatively inert, Hytrast should excite little in the way of reaction in the bronchi. In mice, oral feeding produces evidence neither of acute nor of chronic toxicity. Both in experimental animals and in clinical practice elimination from the bronchi is rapid, two to seven days, and inflammatory or granulomatous reaction has been observed neither in experimental animals nor clinically in operative specimens removed after bronchography with Hytrast.

Hytrast was used as the bronchographic medium in 50 consecutive investigations. In children the quantity of contrast used was calculated from the age of the patient plus $2 \mathrm{ml}$. for each side ; in adults 15 to $20 \mathrm{ml}$. was used for each side.

Five patients in the series of 50 had previously been submitted to bilateral bronchography and required repetition of this investigation. In four, the previous examination had been with Dionosil Oily and in one with sulphanilamide powder in Lipiodol. In all five, good bronchograms were achieved, and in all, contrast was improved in

${ }^{1}$ Supplied by Bengue \& Co., Ltd. 


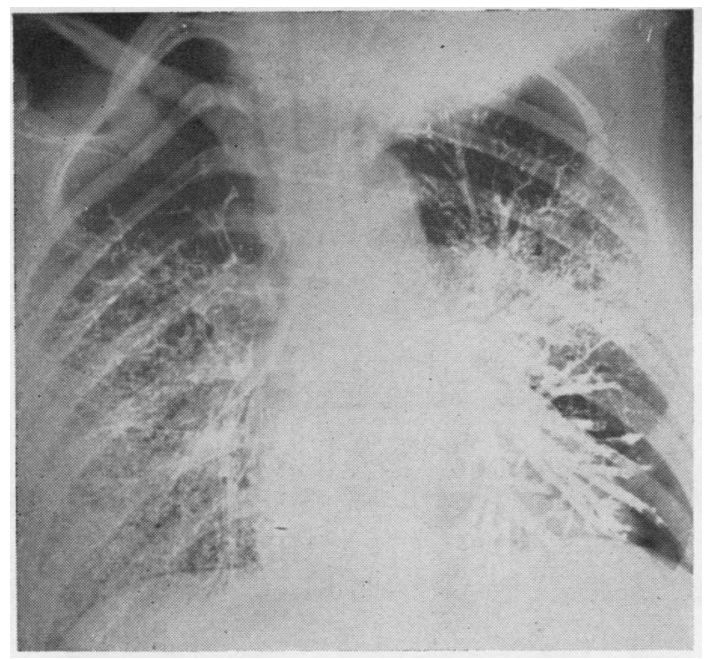

FIG. 1. Bilateral antero-posterior bronchogram made with Dionosil Oily in 1960 for a girl then aged 14 years who was shown to have left basal and lingular bronchiectasis. The bronchiectatic segments werc resected.

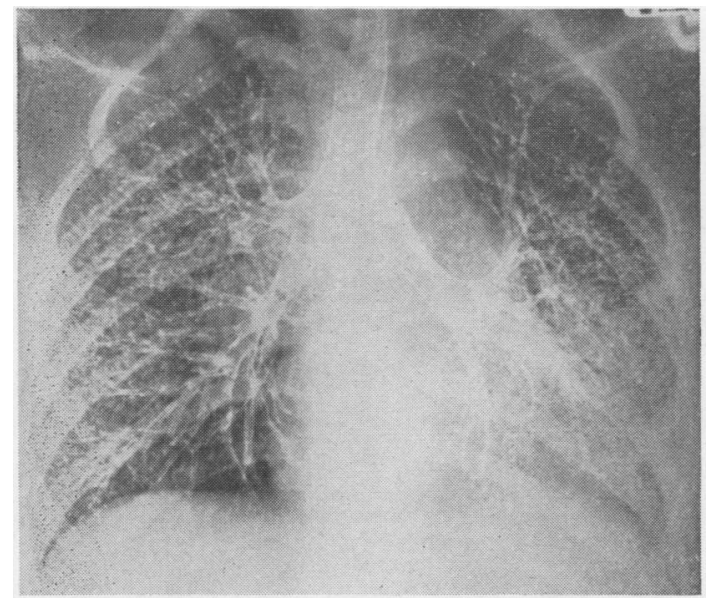

FIG. 2. Routine post-operative bilateral antero-posterior bronchogram made with Dionosil Oily a year later for the girl whose pre-operative radiograph is shown as Fig. 1.

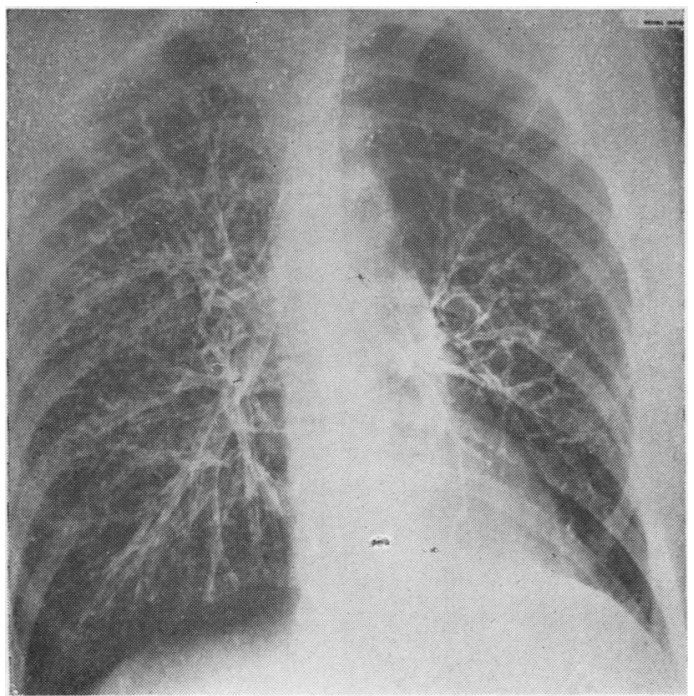

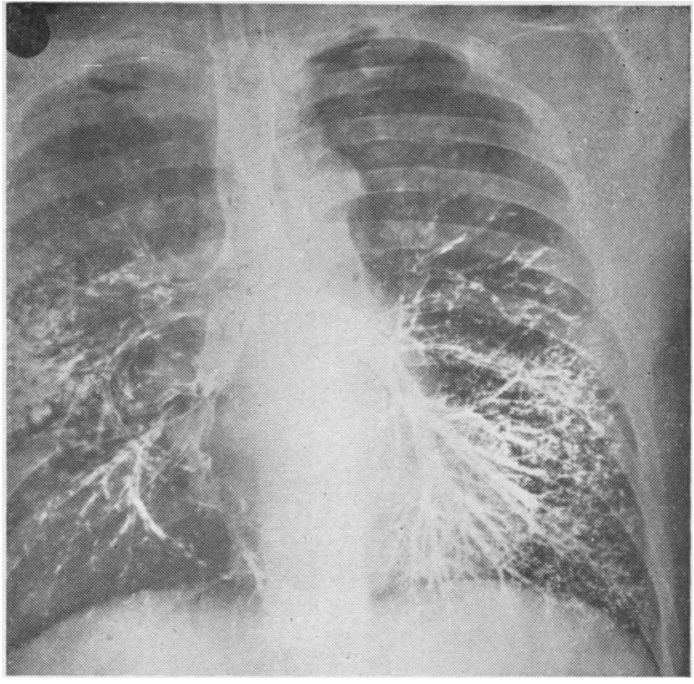

FIG. 4. Bilateral antero-posterior bronchogram made in 1954 for an adult male patient with haemoptysis. The contrast medium was Lipiodol with sulphanilamide powder.

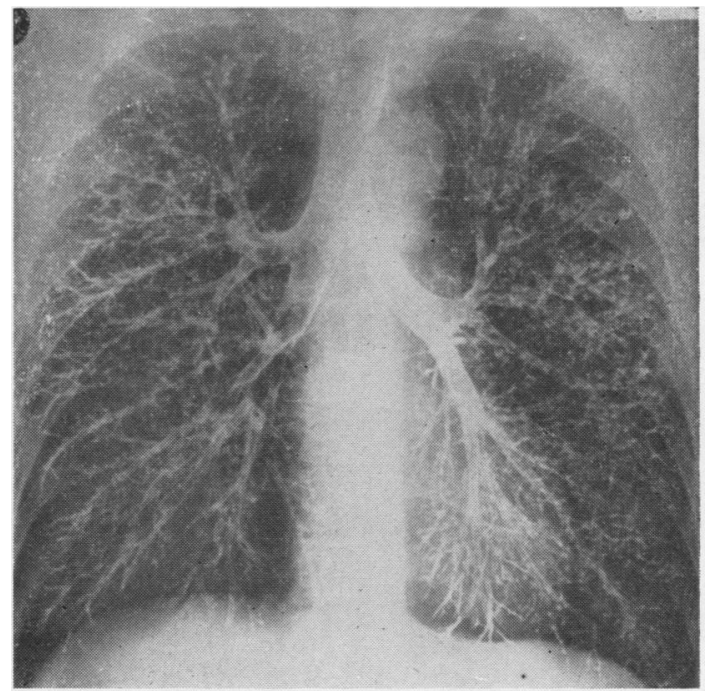

FIG. 5. In the patient whose earlier bronchogram is shown as Fig. 4, haemoptysis recurred in 1963 and bronchography was repeated. Here the contrast medium is Hytrast. Peripheral filling and contrast are good. In this radiograph there are reflected improvements in both bronchographic technique and radiographic contrast. The bronchogram shown as Fig. 4 was acceptable at the time when it was made and would now, 10 years later, be entirely unacceptable.

FIG. 3. Bilateral antero-posterior bronchogram made in 1963 for the girl whose earlier films are shown as Figs. 1 and 2. This further bronchogram was prompted by the complaint of a single small haemoptysis, for which no cause was found at bronchoscopy. Hytrast was used for the bronchogram. Contrast is improved in comparison with the two earlier radiographs; there is no alveolar filling; right supra-phrenic filling is deficient. 


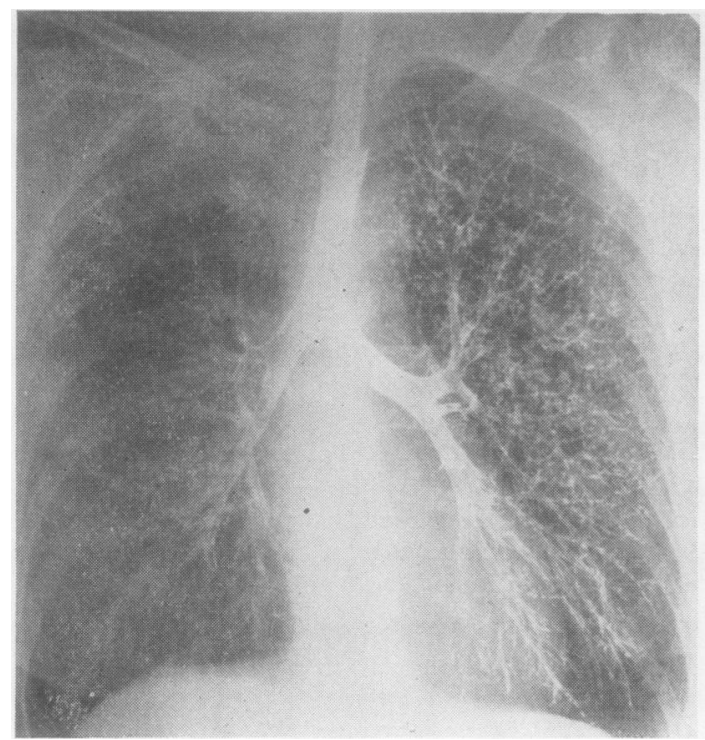

FIG. 6. In this bronchogram the contrast medium used on the right was Dionosil Oily and that used on the left was Hytrast. The right bronchogram was made first and this radiograph was the last of four in this patient, so that the contrast medium in the right lung has been distally propelled by five pulmonary insufflations for the right bronchogram and 10 for the left.

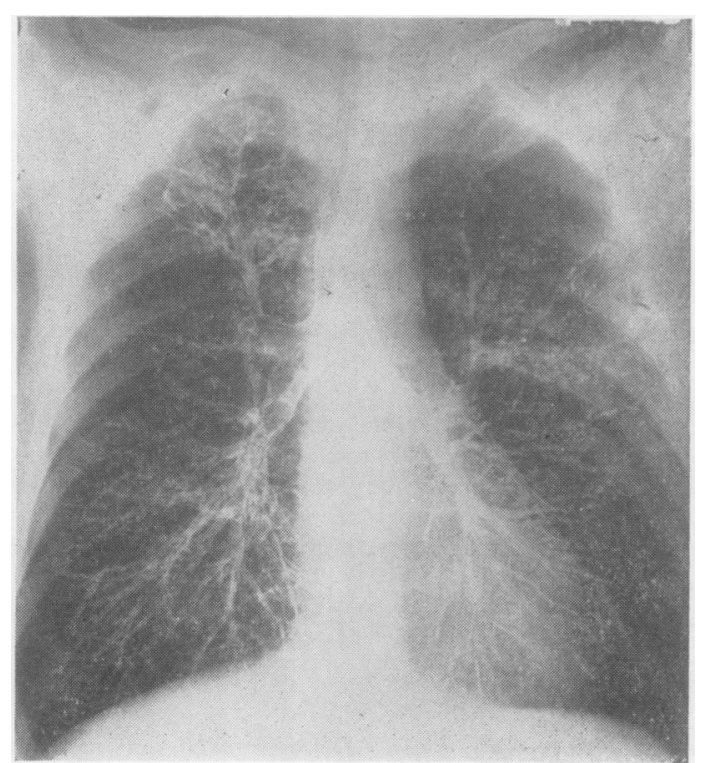

FIG. 7

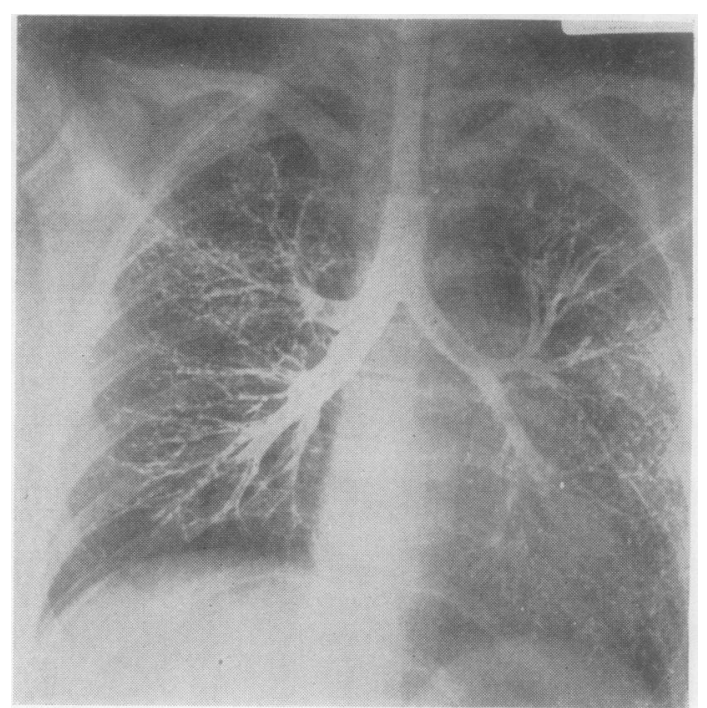

FIG. 8

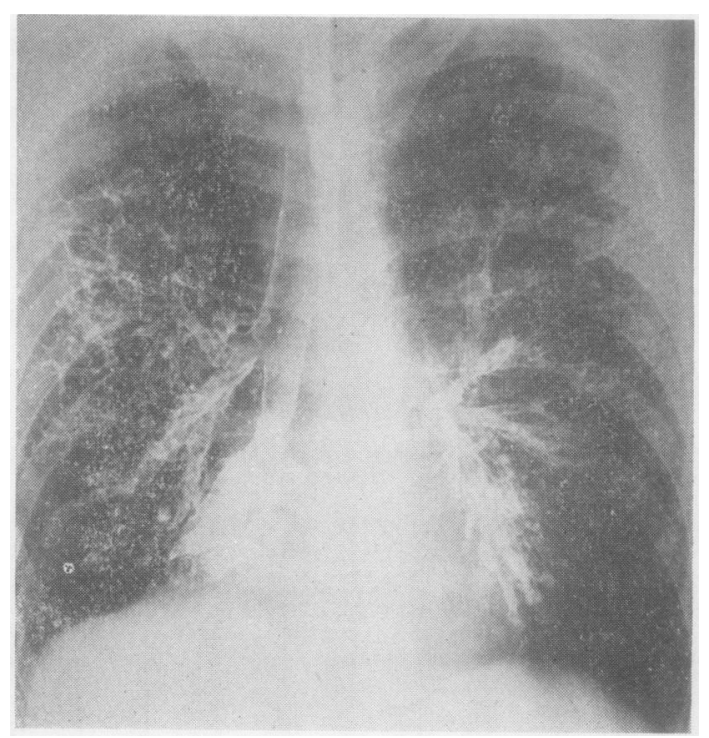

FIG. 9

FIGS. 7, 8, and 9. In these bronchograms the contrast medium used on the right was Hytrast and that on the left Dionosil Oily. The right side was filled first, and, despite 15 pulmonary inflations by the time these films were made, there is no alveolar filling on the right. In Fig. 7 the right posterior segmental bronchus is bronchiectatic. 
comparison with previous bronchograms (Figs. 1 to 5).

In 20 patients, Hytrast and Dionosil Oily were compared, in 10 by using Dionosil for the right bronchogram and Hytrast for the left, and in 10 by using Hytrast for the right and Dionosil for the left. In every instance the right bronchogram was made before the left. It was realized that residual contrast medium in the trachea would be carried into the left bronchi so that a small quantity of whichever contrast medium was first used would be mixed with the contrast medium used for the left bronchogram. In all 20 bronchograms Hytrast, whether used for the right or the left side, produced a bronchogram in which contrast was better, alveolar filling was absent, and which was generally more pleasing, probably because the bronchi were outlined rather than filled. The capacity of Hytrast to outline rather than fill bronchi is attributed to the viscosity of the excipient (carboxymethylcellulose) (Figs. 6 to 9).

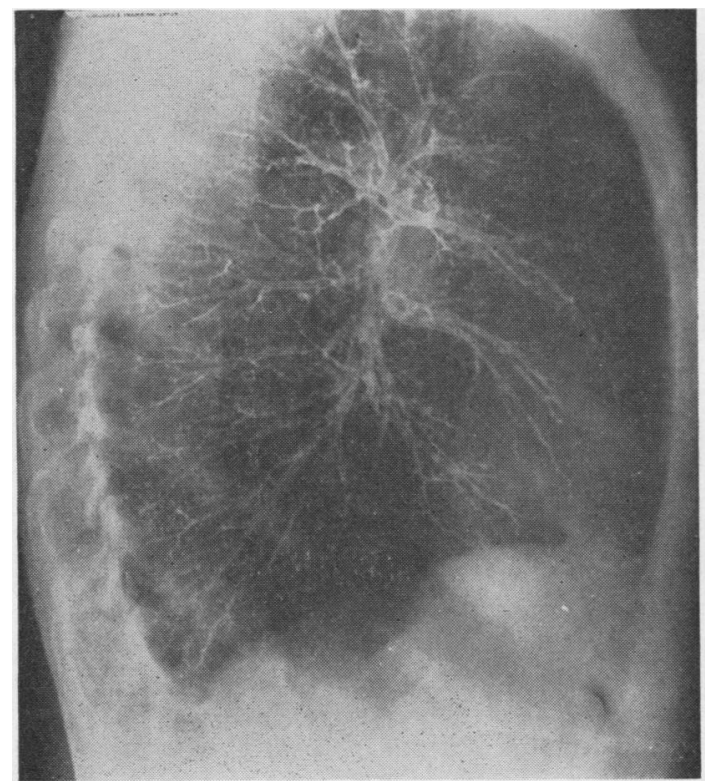

FIG. 10

FIGS. 10, 11, and 12. Right lateral, antero-posterior, and left oblique views; a complete bronchogram made with Hytrast to which sterile water had been added in the proportion $20 \mathrm{ml}$. Hytrast: $1 \mathrm{ml}$. water. Peripheral filling of the bronchi witnout alveolar filling is good and contrast is satisfactory. Filling is uniform throughout the lung fields, and this is a feature of bronchography by insufflation under general anaesthesia with a relaxant. In patients with normal bronchi this is the sort of bronchogram which can be made routinely.
In some of the earlier bronchograms made with Hytrast peripheral bronchi were incompletely outlined. This defect was quickly remedied by the anaesthetist inflating the lungs 10 times for each side as opposed to five times for a bronchogram

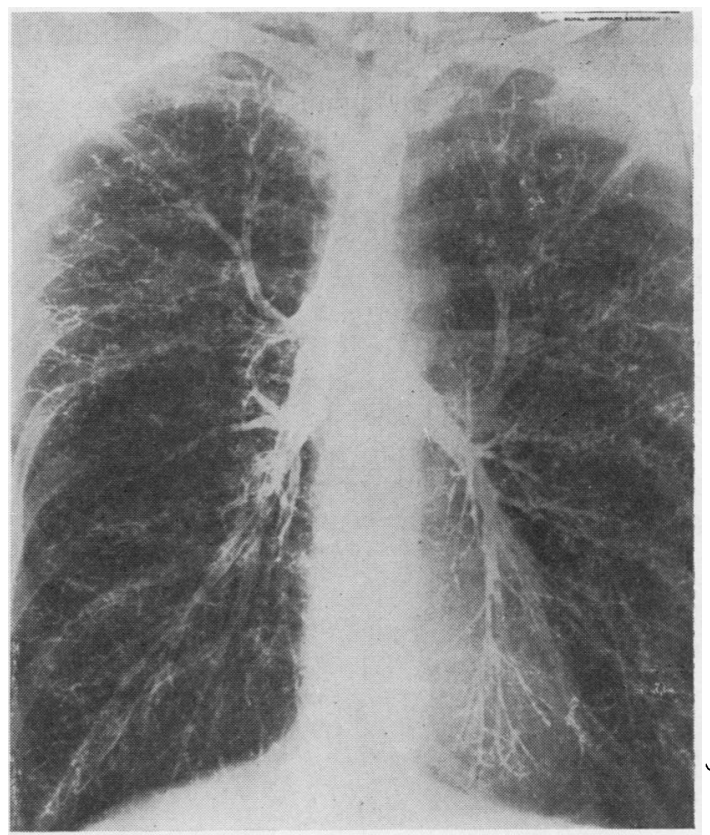

FIG. 11

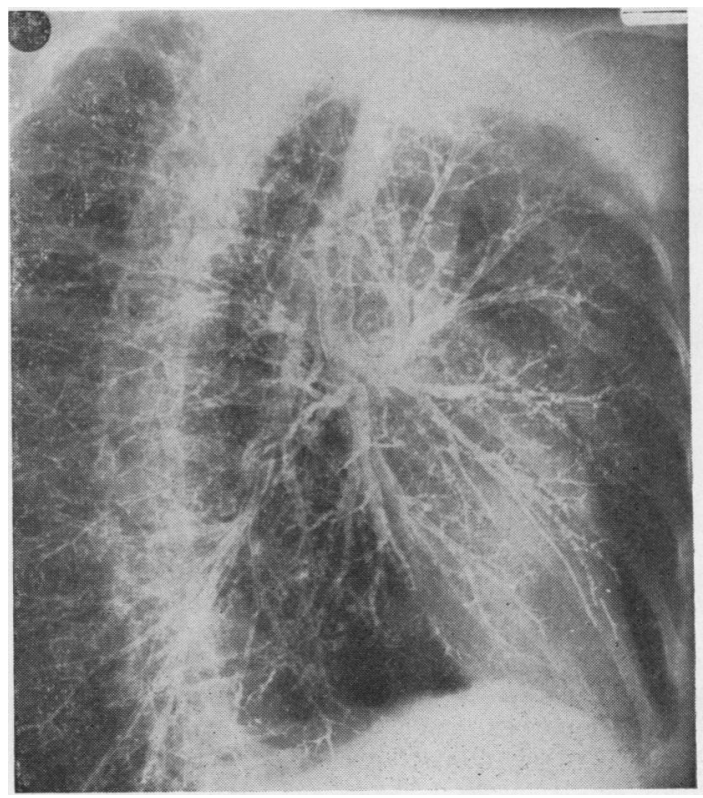

FIG. 12 
with Dionosil Oily. If $1 \mathrm{ml}$. of sterile water is added to each $20 \mathrm{ml}$. of Hytrast a bronchogram of excellent contrast without alveolar filling is achieved with inflation of the lungs seven times for each side. Consistently good peripheral filling without loss of contrast was demonstrated by making 10 bronchograms with Hytrast diluted in the ratio $20 \mathrm{ml}$. Hytrast: $1 \mathrm{ml}$. water (Figs. 10 to 12). In a further 10 patients Hytrast alone was compared with Hytrast diluted in the previously mentioned ratio with water; in five of these 10 , undiluted Hytrast was used on the right and the diluted suspension on the left, and in the other five this relation was reversed. That some of the contrast used for the right bronchogram would remain in the trachea and be carried into the left side when the left bronchogram was made was again realized, but in all 10 bronchograms it was clear that with inflation of the lungs seven times for each side a more complete bronchogram with good peripheral filling was achieved without loss of contrast with the diluted Hytrast (Figs. 13 and 14). Dilution of Hytrast has now been adopted

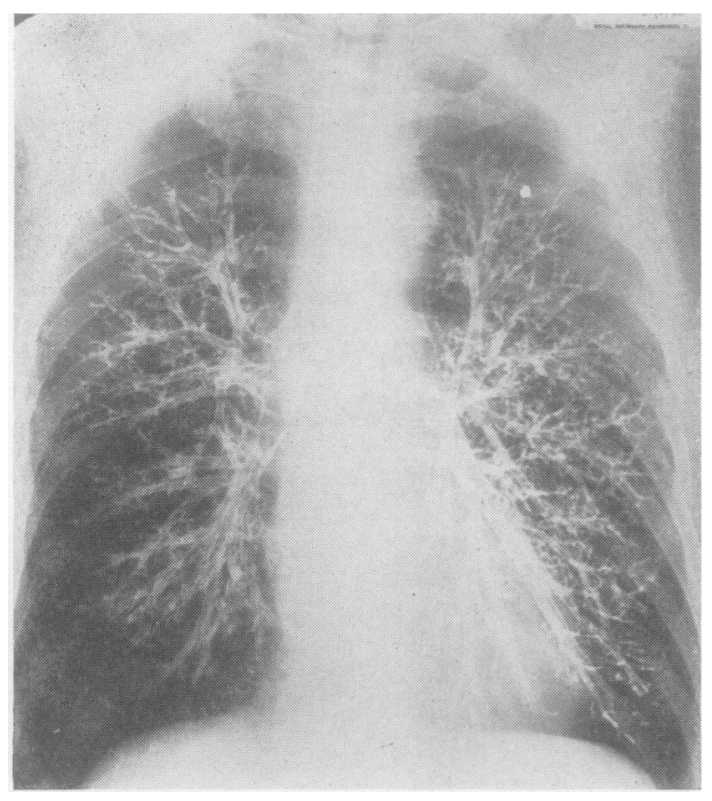

FIG. 13. In this bronchogram Hytrast was used on the right, and on the left Hytrast diluted $20 \mathrm{ml} .: 1 \mathrm{ml}$. water. The right side was filled first. The lungs were inflated seven times. Peripheral filling is improved by the dilution of the Hytrast on the left, without loss of contrast. The improviment in filling on the left is the more obvious when it is remembered that this radiograph was the last of four to be made, so that the right lung has been inflated 14 times with contrast medium in its bronchi.

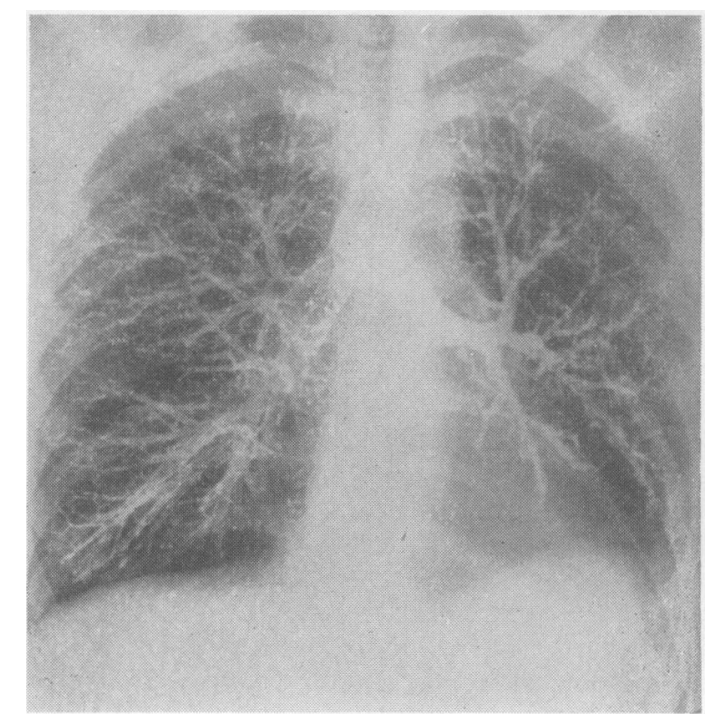

FIG. 14. In this bronchogram dilute Hytrast was used on the right and undiluted Hytrast on the left; the right side was filled first. It is again clear that peripheral filling is improved by dilution of the Hytrast without loss of contrast.

routinely since it shortens the duration of the procedure and decreases the number of relatively forceful inflations of the lungs required for a good bronchogram. Dilution may be unnecessary when bronchograms are made under local anaesthesia with an aspiration rather than an insufflation technique. When Hytrast was diluted in the ratio $20 \mathrm{ml}$. Hytrast:2 ml. water, contrast was diminished and alveolar filling was produced with inflation of the lungs five times for each side. This was demonstrated in five bronchograms (Fig. 15).

In 35 of the 50 patients there were traces of Hytrast visible on chest radiographs made 48 hours after bronchography, and in 15 there was no trace. Of the 35 , all but five had clear chest films four days after bronchography. In one patient with gross bilateral bronchiectasis Hytrast was present in a chest radiograph made nine days after bronchography but had disappeared at 27 days. It may have disappeared earlier, but it was inconvenient for the patient to attend for chest radiography between the ninth and the twentyseventh days after bronchography. Hytrast had cleared in the remaining four patients by the seventh day after bronchography.

In none of the 50 patients was there evidence of bronchial irritation as a consequence of bronchography during a minimum of three days of observation after the investigation. Pyrexia, never higher than $100^{\circ} \mathrm{F}$., was observed in 12 of the 


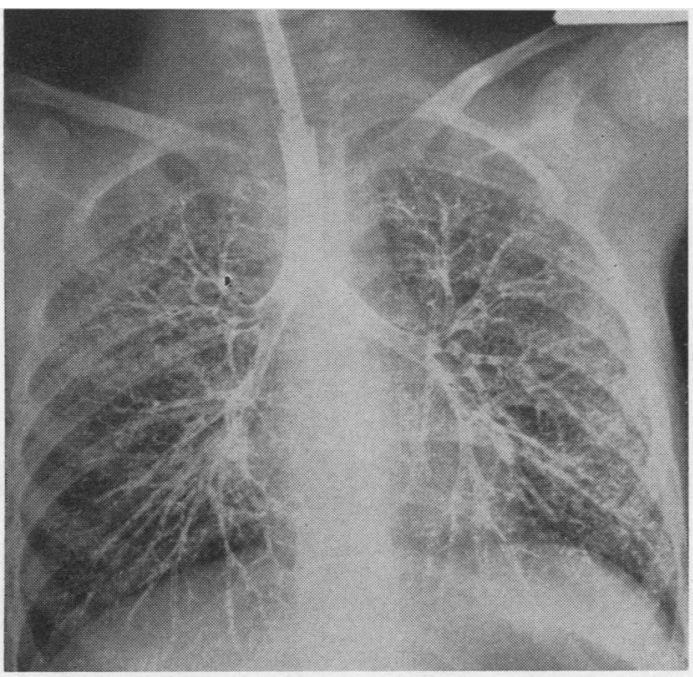

FIG. 15. Bilateral antero-posterior bronchogram made with Hytrast to which sterile water had been added in the ratio $20 \mathrm{ml}$. Hytrast: $2 \mathrm{ml}$. water. Contrast is less good and there is alveolar filling. The lungs were inflated five times.

50 patients on the first or second day after bronchography; none had fever after the third day and none was disturbed. In four patients pulmonary resection followed bronchography, in three less than two weeks after bronchography, and in one four weeks after bronchography; in none of the operative specimens was there evidence of inflammatory or granulomatous reaction to the contrast medium.

\section{SUMMARY}

Hytrast, blown into the bronchi, is recommended as a contrast medium for bronchography following its use in 50 patients in whom bilateral bronchograms were made under general anaesthesia with a relaxant.

\section{REFERENCES}

Burrascano, J. J. (1955). Visciodol in bronchography. Sea View Hosp. Bull., 15, 149.

Dormer, B. A. (1940). Personal communication.

Jackson, C. (1918). The bronchial tree: its study by insufflation of opaque substances in the living. Amer. J. Roentgenol., 5, 454.

Johnson, P. M., and Irwin, G. L. (1959). An evaluation of the pharmacological hazards resulting from use of "Visciodol " in bronchography. Radiology, 72, 816.

le Roux, B. T. (1962). The bronchial anatomy of the left upper lobe J. thorac. Surg., 44, 216.

- and Masson, A. (1962). Bronchography under general anaesthesia. Brit. J. Anaesth., 34, 200.

Sicard, J. A., and Forestier, J. A. (1922). Méthode générale d'exploration radiologique par l'huile iodée (lipiodol). Bull. Soc. méd. Hôp Paris, 46, 463. 